\title{
Recent Developments in the CONRAD Code regarding Experimental Corrections
}

\author{
P. Archier ${ }^{1, a}$, C. De Saint Jean', S. Kopecky², O. Litaize, G. Noguère', P. Schillebeeckx², \\ K. Volev ${ }^{2}$ \\ ${ }^{1}$ CEA, DEN, DER, SPRC, LEPh, Cadarache, F-13108 Saint-Paul-lez-Durance, France \\ ${ }^{2}$ EC-JRC-IRMM, Retieseweg, 2440 Geel, Belgium
}

\begin{abstract}
The CONRAD code is an object-oriented software tool developed at CEA Cadarache since 2005 to deal with problems arising during the evaluation process (data assimilation and analysis, physical modelling, propagation of uncertainties...).

This paper will present recent developments concerning the experimental corrections, which are required when a neutron resonance shape analysis is performed. Several experimental aspects are detailed in this work:

- the possibility to use spectra in energy as well as in time,

- the implementation of both analytical (Chi-Square) and Monte-Carlo resolution functions,

- the sample homogeneity corrections using log-normal distributions.

Each development aspect is illustrated with several examples and comparisons with other resonance analysis codes (SAMMY, REFIT).
\end{abstract}

\section{Introduction}

The CONRAD code [1] is an object-oriented software tool developed at CEA Cadarache since 2005 to deal with problems arising during the evaluation process (data assimilation and analysis, physical modelling, propagation of uncertainties...).

This paper presents recent developments concerning the experimental corrections, which are required for a neutron resonance shape analysis. First, we will explain in Section 2 how CONRAD deals with different types of measurement, which can be provided by users either in energy or in timeOf-flight (ToF). In Section 3, we will detail several resolution functions currently implemented and their validations with other resonance analysis codes such as SAMMY [2] and REFIT [3]. Section 4 presents the sample homogeneity corrections required when evaluators want to analyse experiments with thin powder samples, such as $\mathrm{U} / \mathrm{Pu}$ oxydes.

\section{Time-Of-Flight Experimental Parameters}

We introduced in the code two parameters related to ToF experiments: the well-known flight path $F P$ and the initial delay/time offset $t_{0}$. From now on, if the flight path is provided in the input, CONRAD

ae-mail: pascal.archier@cea.fr 
works in time instead of energy. For instance, if measurements in energy and the flight path are given, the code will first convert the energy grid back in ToF, with Eq. (1), and only then it will do the analysis.

$$
t_{n}[\mathrm{~ns}] \simeq 72298 \cdot \frac{F P[\mathrm{~m}]}{\sqrt{E_{n}[\mathrm{eV}]}}-t_{0}[\mathrm{~ns}]
$$

Since the flight path is a parameter that can be fitted, we prepared a dummy experiment $\left({ }^{113} \mathrm{Cd}\right.$ transmission) where the flight path has been changed to a wrong value $F P=28 \mathrm{~m}$ (instead of the original value: $26.464 \mathrm{~m}$ ). Figure 1 shows the calculated transmissions before the adjustment of the $F P$, where the data are obviously shifted, and the calculated transmissions after the fit which are in excellent agreement with the data.

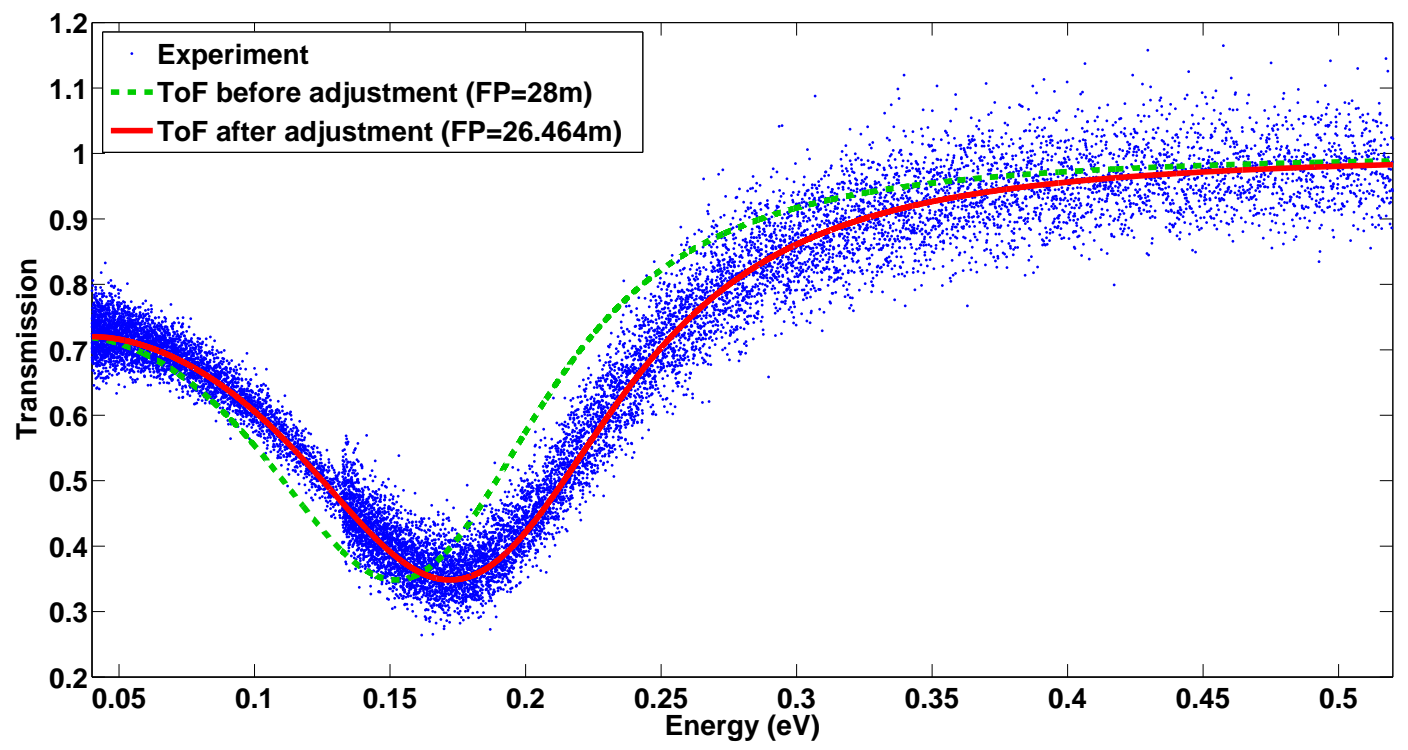

Figure 1. ${ }^{113} \mathrm{Cd}$ transmissions before and after adjustement of the flight path parameter

\section{Resolution Functions}

Resolution function broadening is necessary for most of experimental data analysis [4]. Actually, the true observables can be written respectively for transmissions and capture yields:

$$
\begin{aligned}
& T_{e f f}(E)=\int R\left(E^{\prime}, E\right) T(E) d E^{\prime} \\
& Y_{e f f}(E)=\int R\left(E^{\prime}, E\right) Y(E) d E^{\prime}
\end{aligned}
$$

where $R\left(E^{\prime}, E\right) d E^{\prime}$ stands for the resolution function, i.e. the probability that an event observed at an energy $E$ (or time $t$ ) occured in reality at an energy $E^{\prime}$ in $d E^{\prime}$. 
These deviations in the Time-Of-Flight measurements are mainly due to:

- the accelerator burst width $t_{b}$

- the time channel width $t_{c}$

- the target-moderator assembly $L_{m o d}$

- the Lithium glass detector $L_{d e t}$ (for transmission only)

- etc...

Two kinds of resolution functions have been implemented in CONRAD. The first one is analytical: a $\chi^{2}$ with $n$ degrees of freedom (see Eq. (4)), which is commonly used to represent the time distribution in the moderator assembly. This resolution function contains a parameter $\lambda$, depicting the mean free time (or mean free path if $R$ is expressed in distance) of the neutrons in the moderator. At the moment, the $\lambda$ parameter is constant with the incident neutron energy in CONRAD whereas it is energy-dependent in SAMMY and REFIT. The implementation in CONRAD allows the users to choose between a $\chi^{2}$ distribution in time (in nanoseconds) or in distance (in meters).

$$
R(t) \propto\left(\frac{t}{\lambda}\right)^{\left(\frac{n}{2}-1\right)} \cdot \exp \left(-\frac{t}{\lambda}\right)
$$

The second resolution function is a numerical one, also called in SAMMY as UDR or UserDefined Resolution. In this case, the users provide the different distributions (one distribution for a given incident neutron energy) which are calculated with Monte-Carlo simulations of the experimental facility. Those distributions can describe a part (moderator assembly, detector...) or the whole experimental setup. In CONRAD, users can also provide their resolution functions in time or in distance. Furthermore, we choose a log-lin interpolation for neutron energies between two distributions.
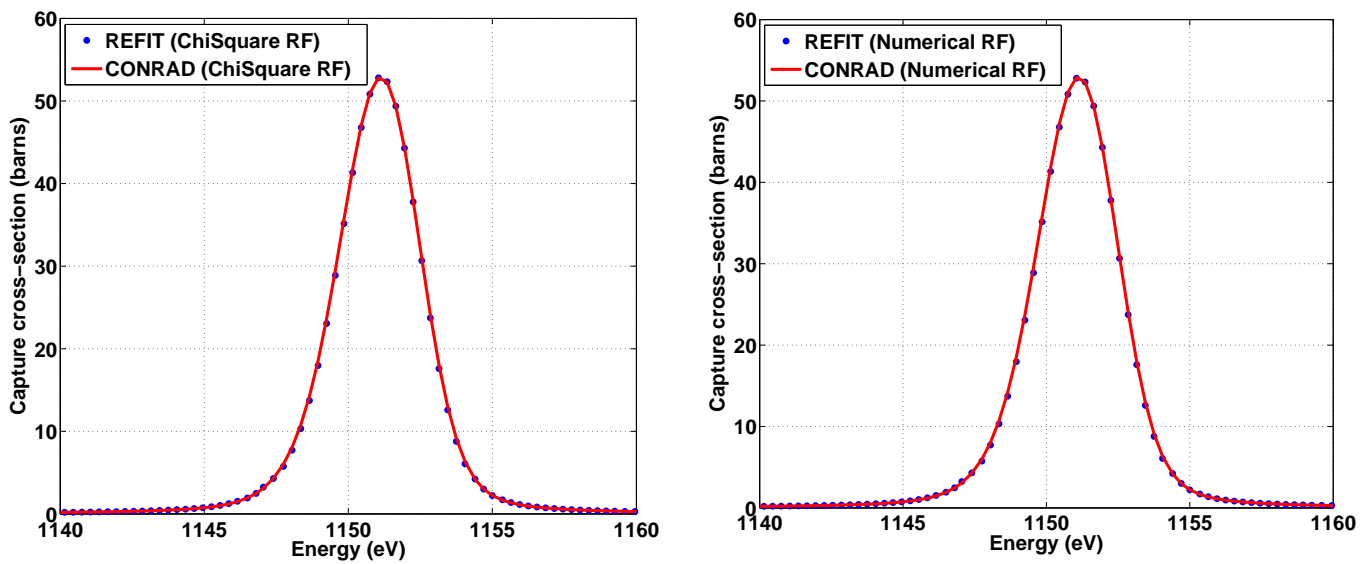

Figure 2. Comparisons of ${ }^{26} \mathrm{Fe}$ capture cross-sections (FGM at $T_{\text {eff }}=293.6 \mathrm{~K}$ ) between CONRAD and REFIT with the analytical ( $\chi^{2}$ with $\lambda=5.83 \mathrm{~mm}$ ) and numerical resolution functions

In order to validate those resolution functions, a test case on ${ }^{56} \mathrm{Fe}$ capture cross section has been prepared: the resonance parameters set is taken from the JEFF-3.1.1 evaluation, the cross sections are broadened with the Free Gas Model (FGM) at an effective temperature of $296.3 \mathrm{~K}$ and the flight path (FP) is set to $28.419 \mathrm{~m}$. Figure 2 shows comparisons between CONRAD and REFIT respectively 
using the $\chi^{2}$ resolution function (with $\lambda=5.83 \mathrm{~mm}$ ) and the numerical resolution function (with distributions coming from the $\chi^{2} \mathrm{RF}$ ). The agreement with the reference code REFIT is excellent. Other comparisons (not displayed for the sake of clarity) have been made versus the SAMMY code and the overall agreement is also very satisfactory.

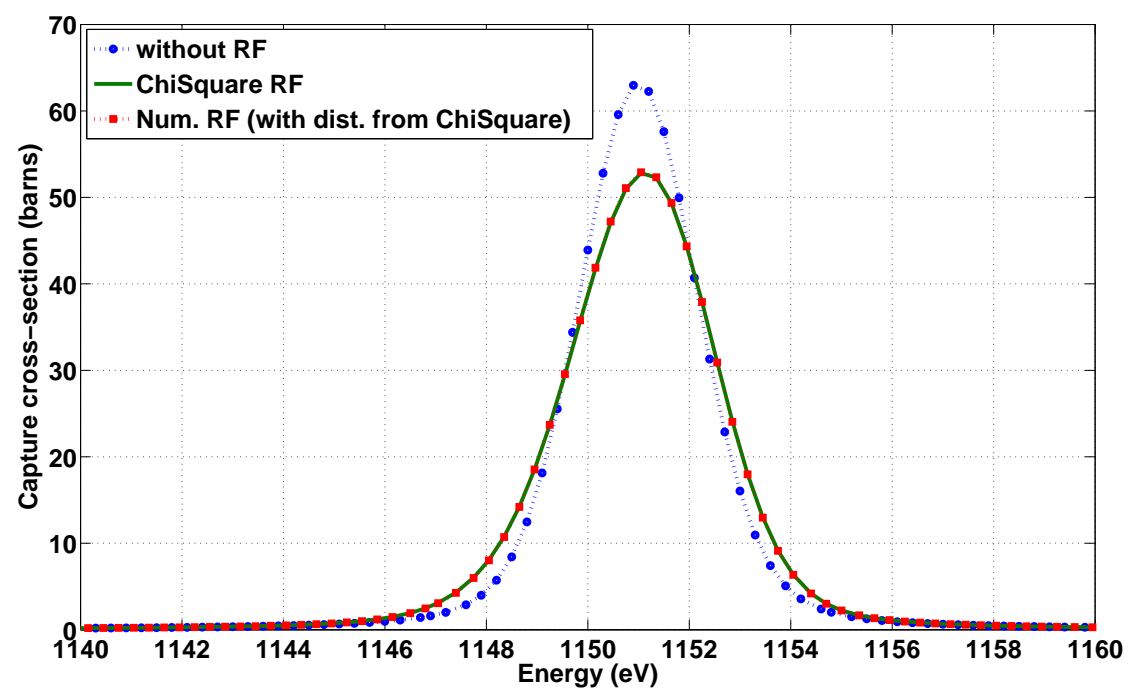

Figure 3. Capture cross-sections from CONRAD without resolution broadening, with the $\chi^{2}$ and with the numerical resolution functions

On Figure 3, we can observe the different capture cross sections calculated with CONRAD without resolution broadening, with the $\chi^{2} \mathrm{RF}$ and with the numerical RF. The impact of the resolution function on the ${ }^{56} \mathrm{Fe}$ resonance is quite large and highlights the importance of resolution-broadening during the resonance shape analysis in this case.

\section{Sample Homogeneities}

Sample homogeneity corrections are required when evaluators want to analyse measurements through samples containing inhomogeneities, commonly found in thin powder samples such as U/Pu oxydes. For instance, those samples can show large porosity (holes) or/and a non-constant thickness. In Ref. [5], S. Kopecky detailed a model to describe the thickness in the sample using a log-normal distribution. This model is available in the REFIT code to account for powder sample inhomogeneities and it has been implemented in CONRAD as well for transmission experiments.

The experimental modeling of the transmission without sample inhomogeneities is given in Eq. (5), where $n$ stands for the thickness (areal density) of the sample (in at./barn).

$$
T(E)=\exp \left[-n \cdot \sigma_{\text {tot }}(E)\right]
$$

In the presence of porosity/holes $p$ (given in \%) in the sample, we obtain Eq. (6) for the transmission, containing two contributions: $p$, where neutrons do not interact with matter (everything goes through) and the rest $(1-p)$, where neutrons transmit through pure and densier matter as detailed on Figure 4. 


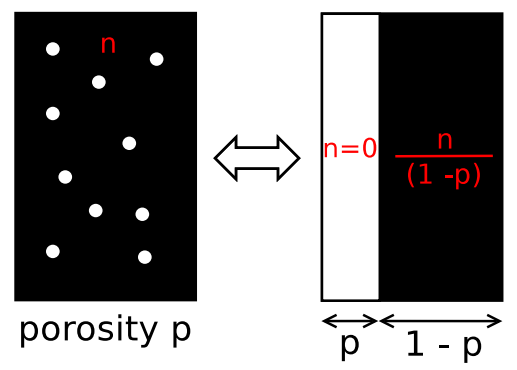

Figure 4. Porosity $p$ in the real sample (left) and model used for transmission (right)

$$
T(E)=(1-p) \cdot \exp \left[-\frac{n}{(1-p)} \cdot \sigma_{t o t}(E)\right]+p
$$

Eq. (7) presents the generalization of the transmission in presence of porosities and using a probability density function $p d f$ (a log-Normal distribution) for the thickness.

$$
T(E)=(1-p) \cdot \int_{0}^{\infty} p d f(x) \exp \left[-\frac{n \cdot x}{(1-p)} \cdot \sigma_{t o t}(E)\right] d x+p
$$

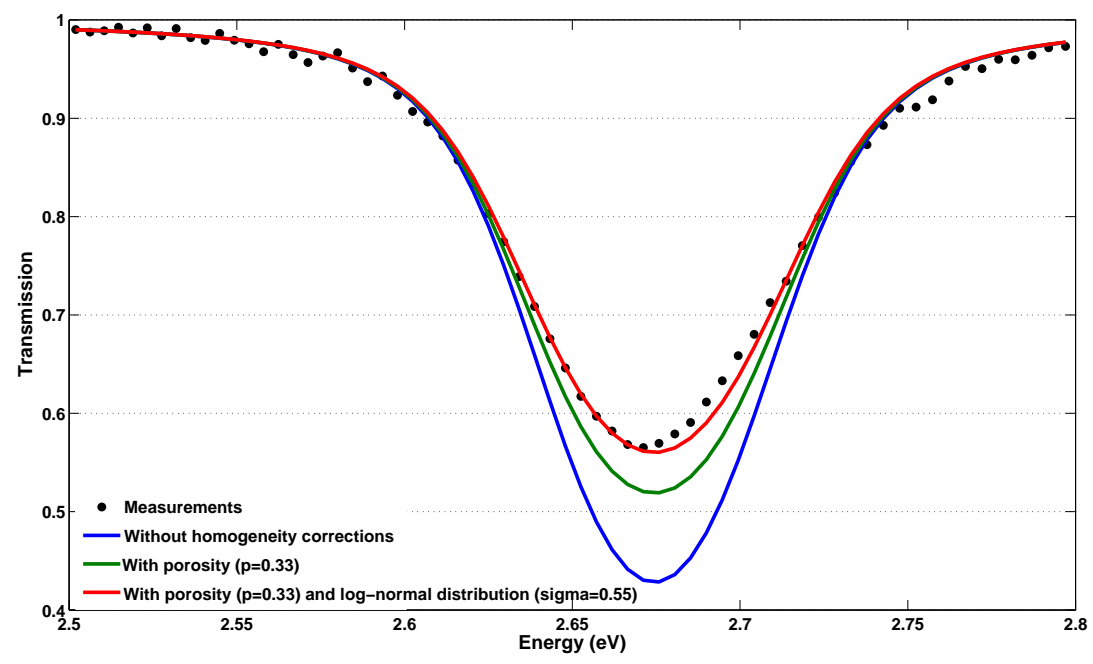

Figure 5. Sample homogeneity corrections on ${ }^{242} \mathrm{Pu}$ transmission experiment

As a validation purpose, we used ${ }^{242} \mathrm{Pu}$ transmission measurements, with a thickness $n=$ $2.5110^{-5}$ at./b. The cross-sections have been Doppler-broadened using the Free-Gas model at an effective temperature of $295.45 \mathrm{~K}$. Calculations have been performed with CONRAD using respectively Eq. (5), (6) and (7) for transmission and results can be seen on Figure 5. First, the calculated transmissions without any kind of homogeneity corrections do not agree well with the experimental data. Using the porosity corrections (with $33 \%$ of holes in the sample), the agreement with the 
measurements improves significantly. At last, the calculated transmissions with the full sample corrections (porosity and log-Normal thickness distribution) are almost able to reproduce correctly the experimental data. Still, on the right wing of the resonance, the agreement is not perfect and should be improved using a Crystal Lattice Model instead of the Free Gas one.

\section{Conclusion}

Several developments in CONRAD focusing on experimental corrections have been carried out. Users can analyse both energy and Time-of-Flight measurements in the same way. Two kinds of resolution functions, analytical $\left(\chi^{2}\right)$ and numerical, have been implemented and validated versus REFIT and SAMMY. Also, sample inhomogeneities corrections for porosity and thickness distribution in the case of transmissions have been tested in CONRAD.

In the near future regarding the experimental corrections, the implementation of new analytical resolution functions, such as gaussian distribution, will be considered. Also, we plan to add an energy-dependence for the $\lambda$ parameter used in the $\chi^{2}$ resolution function. Finally, the current Crystal Lattice Model implemented in CONRAD needs to be validated and tested on the ${ }^{242} \mathrm{Pu}$ transmission experiments with inhomogeneities.

\section{Acknowledgements}

This work has been carried out in the framework of the ERINDA project and the CEA/IRMM collaboration. One of the authors (P.A.) would like to thank the IRMM members for the fruitfull discussions and particulary to P. Schillebeeckx, S. Kopecky and K. Volev.

\section{References}

[1] C. De Saint Jean, B. Habert, O. Litaize, G. Noguère, C. Suteau, Status of CONRAD, a nuclear reaction analysis tool, in Proceedings of the International Conference on Nuclear Data for Science and Technology - ND2007, edited by EDP Sciences (Nice, France, 2007).

[2] N.M. Larson, Updated Users: Guide for SAMMY: Multilevel R-Matrix Fits to Neutron Data Using Bayes Equations, Oak Ridge National Laboratory Tech. Report, ORNL/TM-9179/R8, (2008).

[3] M.C. Moxon, T.C. Ware, C.J. Dean, REFIT-2009 A Least-Square Fitting Program for Resonance Analysis of Neutron Transmission, Capture, Fission and Scattering Data, Users' Guide for REFIT2009-10, Tech. rep., UKNSF (2010).

[4] F.H. Fröhner, Evaluation and Analysis of Nuclear Resonance Data, OECD/NEA JEFF Report 18 (2000).

[5] S. Kopecky, P. Siegler, A. Moens, Low energy transmission measurements of ${ }^{240,242}$ Pu at GELINA and their impact on the capture width, in Proceedings of the International Conference on Nuclear Data for Science and Technology - ND2007, edited by EDP Sciences (Nice, France, 2007). 